\title{
Metabolic fate (absorption, $\beta$-oxidation and deposition) of long-chain $n-3$ fatty acids is affected by sex and by the oil source (krill oil or fish oil) in the rat
}

\author{
Samaneh Ghasemifard ${ }^{1 *}$, Karen Hermon ${ }^{2}$, Giovanni M. Turchini ${ }^{2}$ and Andrew J. Sinclair ${ }^{1}$ \\ ${ }^{1}$ School of Medicine, Deakin University, Geelong, VIC 3216, Australia \\ ${ }^{2}$ School of Life and Environmental Sciences, Deakin University, Warmambool, VIC 3280, Australia \\ (Submitted 1 March 2015 - Final revision received 28 May 2015 - Accepted 4 June 2015 - First published online 3 August 2015)
}

The effects of krill oil as an alternative source of $n-3$ long-chain PUFA have been investigated recently. There are conflicting results from the few available studies comparing fish oil and krill oil. The aim of this study was to compare the bioavailability and metabolic fate (absorption, $\beta$-oxidation and tissue deposition) of $n-3$ fatty acids originating from krill oil (phospholipid-rich) or fish oil (TAG-rich) in rats of both sexes using the whole-body fatty acid balance method. Sprague-Dawley rats (thirty-six male, thirty-six female) were randomly assigned to be fed either a krill oil diet (EPA + DHA + DPA $=1.38 \mathrm{mg} / \mathrm{g}$ of diet $)$ or a fish oil diet $(\mathrm{EPA}+\mathrm{DHA}+\mathrm{DPA}=1.61 \mathrm{mg} / \mathrm{g}$ of diet) to constant ration for 6 weeks. The faeces, whole body and individual tissues were analysed for fatty acid content. Absorption of fatty acids was significantly greater in female rats and was only minimally affected by the oil type. It was estimated that most of EPA $(>90 \%)$ and more than half of DHA $(>60 \%)$ were $\beta$-oxidised in both diet groups. Most of the DPA was $\beta$-oxidised ( 57 and $67 \%$ for female and male rats, respectively) in the fish oil group; however, for the krill oil group, the majority of DPA was deposited (82-83\%). There was a significantly greater deposition of DPA and DHA in rats fed krill oil compared with those fed fish oil, not due to a difference in bioavailability (absorption) but rather due to a difference in metabolic fate (anabolism $v$. catabolism).

Key words: Krill oil: EPA: DHA: DPA: Metabolic fate: Tissue deposition: Sex

The beneficial effects of marine oils such as fish oil and krill oil on human health and well-being are mostly attributed to the high content of $n$-3 long-chain PUFA (LC-PUFA), predominantly EPA (20:5n-3), DPA $(22: 5 n-3)$ and DHA $(22: 6 n-3)^{(1-3)}$. As a result of these beneficial effects, there have been recommendations that individuals should increase their daily intake of $n-3$ LCPUFA. Consequently, the use of food supplements in the form of fish oil capsules has become popular. In the last few years, krill oil has entered the market as an alternative source of $n-3$ fatty acids to traditional fish oil. It has been claimed by various industry sources, with no clearly documented evidence, that $n-3$ LC-PUFA from krill oil are much more 'bioavailable' than those from fish oil. This has been attributed to differences in molecular structure and antioxidant content between these two marine sources. In fish oil, the $n-3$ LC-PUFA are mostly esterified in TAG, whereas in krill oil the fatty acids are largely found in phospholipids (PL), TAG and NEFA ${ }^{(4)}$. In addition, krill oil contains various antioxidants including astaxanthin and flavonoids, which are not present in fish $\mathrm{Oil}^{(4)}$.

There are several available studies comparing the effects of fish oil and krill oil in animals ${ }^{(5-7)}$ and humans ${ }^{(8-12)}$. In general terms, the data do not show a consistently higher blood or tissue levels of EPA or DHA in the krill oil-fed groups compared with the fish oil-fed groups. Furthermore, a fairly common limitation of these studies was the failure to have identical doses of $n$ - 3 LC-PUFA between treatments. In addition, little attention has been thus far focused on possible confounding effects originating by sexspecific responses to dietary $n$-3 LC-PUFA intake, despite evidence of more efficient $n$-3 LC-PUFA metabolism in women compared with men ${ }^{(13-15)}$. Accordingly, several rat studies examined the expression of enzymes involved in the biosynthesis of DHA from shorter-chain $n$ - 3 fatty acids, finding that female rats replete their DHA status more readily than males, likely due to a higher expression of liver $\Delta 5$ - and $\Delta 6$-desaturase enzymes ${ }^{(16-18)}$. Nevertheless, there is a lack of information relative to possible sex differences with respect to $n$-3 LC-PUFA bioavailability and deposition when provided in the diet in different chemical forms.

Salem \& Kuratko ${ }^{(19)}$ recently reviewed studies comparing the 'bioavailability' of fish oil and krill oil. They concluded that there was no evidence for greater 'bioavailability' of $n-3$ LCPUFA from krill oil $v$. fish oil and that more carefully controlled human trials must be performed to establish their relative efficacies after chronic administration. Following this review, Ghasemifard et al. ${ }^{(20)}$ critically reviewed the methodology used in existing studies on the 'bioavailability' of $n$ - 3 LC-PUFA in different forms, and highlighted that the mixed and inconclusive results currently available were likely due to the implementation of different, and often not appropriate, methodologies.

Abbreviations: BW, body weight; PL, phospholipids; LC-PUFA, long-chain PUFA.

*Corresponding author: S. Ghasemifard, fax +613522 72170, email sghasemi@deakin.edu.au 
As recently discussed in the latter study ${ }^{(20)}$, in pharmacology, 'bioavailability' refers to the measurement of the extent to which a drug reaches the systemic circulation, and is therefore a well-defined and standardised term ${ }^{(21)}$. Nevertheless, when used in nutrition science, there is no agreed definition for the term 'bioavailability', and it has become a sort of generic term used to estimate how much of a given nutrient is retained in the body. As argued by Ghasemifard et al. ${ }^{(20)}$, in essence, 'bioavailability' is not different from the term 'digestibility', which is a well-defined term and its methods of evaluation are agreed and standardised. When it comes to $n$-3 LC-PUFA and related studies, 'bioavailability' is often used referring to the blood levels of $n$ - 3 LC-PUFA achieved after consuming an $n-3$ LC-PUFA dose, but rarely has the excretion of $n$-3 LC-PUFA been measured. As $n$-3 LC-PUFA levels in blood are directly affected by a number of complex processes (including digestion, absorption and metabolism) and are also influenced by other external factors (such as the fat content of the diet), it has been suggested that the blood level is not a reliable measure of $n$-3 LC-PUFA 'bioavailability'. Therefore, in the present study, we decided to minimise the use and reference to the term 'bioavailability' and more specifically focus on absorption, retention and metabolic fate.

The whole-body fatty acid balance $\operatorname{method}^{(22)}$ is rapidly gaining popularity and has been successfully implemented in a variety of animal species. It is envisaged that it could be a more 'efficient', more reliable and a simple method to estimate and quantify an organism's overall capacity to metabolise fatty acids, within the context of an integrated system.

Therefore, the aim of the present study was to compare the metabolic fate (absorption, $\beta$-oxidation and tissue deposition) of dietary $n$-3 LC-PUFA originating from krill oil (PL-rich) or fish oil (TAG-rich) in male and female rats using the whole-body fatty acid balance method.

\section{Methods \\ Diet and study design}

This study was performed following the Australian code for the care and use of animals for scientific purposes, and the study was approved by the Deakin University Animal Welfare Committee (G29-2012).

A total of seventy-two 6-week-old healthy and sexually mature male ( $n$ 36) and female ( $n$ 36) Sprague-Dawley rats were purchased from the Animal Resources Centre, Western Australia. Rats were housed in pairs (two rats of the same sex per cage; thirty-six cages in total) and acclimatised for a week on ad libitum normal chow diet. The thirty-six cages were then randomly allocated into six groups of six cages each (three groups per sex). One group of each sex was killed at day 0 for obtaining baseline data. The other four groups were fed two specifically prepared diets (10\% fat by weight; Speciality Feed Company) that contained either krill oil or fish oil (both supplied by Swisse). The experimental design can, therefore, be described as a $2 \times 2$ factorial design, where the factors considered were sex (male $v$. female) and diet (krill oil diet $v$. fish oil diet), and thus giving rise to four treatments: females fed krill oil; females fed fish oil; males fed krill oil; and males fed fish oil. Each treatment group consisted of six replicate cages $(n 6)$, and each cage (the experimental unit) contained a pair of rats of the same sex. The two experimental diets were designed to be isoproteic, isolipidic and to contain equal amounts of EPA+ DHA+DPA, equating to approximately $1.5 \mathrm{mg} / \mathrm{g}$ of the diet. However, the resulting manufactured diets had slightly different total amounts of these fatty acids (Table 1), with the krill oil diet recording $\mathrm{EPA}+\mathrm{DHA}+\mathrm{DPA}=1.38 \mathrm{mg} / \mathrm{g}$ of the diet and the fish oil diet recording $\mathrm{EPA}+\mathrm{DHA}+\mathrm{DPA}=1.61 \mathrm{mg} / \mathrm{g}$ of diet. The fish oil used contained 88\% TAG, 0.6\% NEFA, $4.8 \%$ DAG, $6.4 \%$ sterols and $0 \cdot 1 \% \mathrm{PL}$, whereas the krill oil contained $18 \%$ TAG, $10 \%$ NEFA, $7.4 \%$ sterols and $65 \%$ PL (as determined using an automated TLC scanning instrument; Iatroscan). During the experimentation, animals were fed fixed pre-determined ration, which was adjusted weekly relative to body weight (BW), for 6 weeks. For the computation of the food ration, a preliminary short trial was implemented over a period of 3 weeks, where fifteen rats of similar size were fed ad libitum and the total feed consumption was recorded. Subsequently, using a regression equation comparing BW, the daily ration was estimated as Ration $(\% \mathrm{BW})=-0.0283 \mathrm{BW}+16 \cdot 6$. Throughout the 6-week period, the rats were weighed (twice per week), and the faeces were collected every day during the last 2 weeks. After the 6-week period, the rats were humanely killed with $\mathrm{CO}_{2}$ overdose. Six rats from each group were used for the analysis of whole-body lipids (one from each cage of two rats), and six rats were used for the analysis of the individual tissues (the other rat from the cage housing pairs of rats of the same sex). The collected faeces samples, whole body and tissues (liver, heart, white gastrocnemius muscle and perirenal adipose tissue) were analysed for fatty acid content.

\section{Lipid analysis}

The whole body, tissues and faeces samples were homogenised, and lipids were extracted using dichloromethane:methanol (2:1), a modification of the method described by Folch et $a l .{ }^{(23)}$. Fatty acids derived from the lipids were methylated using an acidcatalysed transmethylation $^{(24)}$. In brief, an aliquot of fatty acids derived from the lipids plus a known amount of internal standard of tricosanoic acid (C23 : 0>99\%; Nu-Chek Prep Inc.) were

Table 1. Dietary proximate composition and selected fatty acid concentrations $(\mathrm{mg} / \mathrm{g}$ of diet)

(Mean values of triplicate analysis)

\begin{tabular}{lcc}
\hline Composition (mg/g of diet) & Fish oil diet & Krill oil diet \\
\hline Protein & 194 & 194 \\
Fibre & 84 & 84 \\
Fat & 100 & 100 \\
Fatty acid & & \\
$16: 0$ & 38.49 & 36.02 \\
$18: 0$ & 3.75 & 3.41 \\
$18: 1 n-9$ & 26.91 & 24.31 \\
$18: 2 n-6$ & 7.83 & 7.13 \\
$18: 3 n-3$ (ALA) & 0.49 & 0.41 \\
$18: 4 n-3$ & 0.14 & 0.15 \\
$20: 3 n-3$ & 0 & 0 \\
$20: 5 n-3$ (EPA) & 0.88 & 0.84 \\
$22: 5 n-3$ (DPA) & 0.13 & 0.04 \\
$22: 6 n-3$ (DHA) & 0.59 & 0.51 \\
\hline
\end{tabular}

ALA, $a$-linolenic acid; DPA, docosapentaenoic acid. 
reacted with acetyl chloride/methanol to form fatty acid methyl esters (FAME). The resulting FAME were separated and quantified using an Agilent Technologies 7890A GC system (Agilent Technologies), equipped with a BPX70 capillary column (120 m, $0.25 \mathrm{~mm}$ internal diameter, $0.25 \mu \mathrm{m}$ film thickness; SGE Analytical Science), an Agilent Technologies 7693 autosampler (Agilent Technologies), a split injection system and a flame-ionisation detector using established protocols ${ }^{(25)}$.

\section{Lipid and fatty acid apparent digestibility}

Apparent digestibility of total lipids was measured according to Tou et l. $^{(7)}$ as [(lipid intake-faecal lipid)/(lipid intake)] $\times 100$. Similarly, apparent digestibility of individual fatty acids was measured using the formula [(fatty acid intake-fatty acid excretion in faeces)/(fatty acid intake) $\times 100$. Lipid intake was determined as diet consumed per week $\times$ percentage of lipid in the diet. Likewise, fatty acid intake was determined as diet consumed per week $\times$ percentage of fatty acid in the diet. Faecal lipid and fatty acid contents were determined as total faeces excreted per week (pooled $7 \mathrm{~d}$ faecal samples) $\times$ percentage of lipid and percentage of fatty acids in the faeces, respectively. Rats were housed in pairs, and the faecal lipid data are presented as average digestibility of each rat pair over the last 2 weeks of the trial.

\section{Whole-body fatty acid balance method}

The fatty acid metabolism of rats was determined using the whole-body fatty acid balance method, as conceived and described by Turchini et $a l^{(22)}$, with subsequent developments ${ }^{(26)}$. In brief, the first step of the method required that the net appearance or disappearance of each individual fatty acid be determined by the difference between total fatty acid accumulation (final fatty acid content-initial fatty acid content) and the net fatty acid intake (total fatty acid intake-fatty acid excretion in faeces), as initially proposed by Cunnane's group $^{(27-29)}$. Subsequently, after the transformation of data from gram per animal per duration of the trial to moles of fatty acid per gram of BW per day, the following second step involved a series of backwards computations along all the known fatty acid bioconversion pathways ${ }^{(22)}$; therefore, the fate of each individual fatty acid towards bioconversion, $\beta$-oxidation or deposition was determined and quantified. Eventually, data relative to apparent in vivo enzyme activity could be reported as nanomoles of the enzyme's product per gram of BW per day, and the in vivo metabolic fate (absorption, $\beta$-oxidation, bioconversion and tissue deposition) of each dietary fatty acid could be reported as a percentage relative to the dietary intake.

\section{Statistical analysis}

Data analysis was performed using Minitab Statistical Software (version 14; Minitab Inc.). All data are reported as mean values and their standard deviations of six cages per treatment $(n 6$; $N 24$ ). Significant differences between experimental groups were tested using two-way ANOVA, assessing the effects of diets, sex and their interactions. Paired tests were performed using Tukey's test. Statistical significance was considered for $P<0 \cdot 05$.

\section{Results}

\section{Weight of rat whole body and tissues}

There was no significant difference $(P=0.303)$ in the BW of female rats between the two dietary groups during the 6-week period of the experiment. The average BW of animals in fish oil and krill oil groups were 270 (sD 20) and 278 (sD 15) g, respectively, at the end of the experiment. Similarly, there was no significant difference $(P=0.957)$ in the BW of male rats between the two dietary groups during the 6-week period of the experiment. The average BW of animals in fish oil and krill oil groups were 409 (SD 28) and 410 (SD 31) g, respectively, at the end of the experiment. As male rats consumed more diet than female rats, they were significantly heavier (409 (SD 29) v. 274 (sD 18) g, $P=0 \cdot 000)$.

With regard to tissue weight, there was no significant difference in the tissue weights of rats between the two dietary groups during the 6-week period of the experiment. All tissue weights were significantly higher in male rats compared with female rats (online Supplementary Table S1).

\section{Lipid content of rat whole body and tissues}

Whole-body lipid contents ( $\mathrm{mg} / \mathrm{g}$ of wet tissue of whole body) of rats at the conclusion of the study (week 6) were significantly higher compared with the baseline data $(P=0 \cdot 001)$. Female rats had a tendency $(P=0.053)$ towards higher whole-body lipid content compared with male rats. There was no significant difference in the total amount of whole-body lipid content between the two dietary treatments over the 6-week period of the experiment $(P=0.404)$. Furthermore, the interaction between diet and sex was not significant $(P=0 \cdot 269)$.

With regard to the tissue lipid content, both male and female rats had greater liver lipid contents $(\mathrm{mg} / \mathrm{g}$ tissue of wet tissue) over the 6-week period of the experiment compared with the baseline data $(P<0 \cdot 05)$. The total lipid content of the tissues did not differ between diet treatments. The lipid contents of the liver, heart and muscle (mg/g tissue) were significantly higher in female rats compared with male rats $(P<0 \cdot 05$; Table 2$)$.

\section{Lipid and fatty acid apparent digestibility}

The effects of fish oil and krill oil diets on apparent digestibility are shown in Table 3. There were no significant differences observed in total lipid apparent digestibility among the diet or sex groups, with the values exceeding $96 \%$ in each case. However, there was a different pattern for $n-3$ LC-PUFA apparent digestibility, with the digestibility ranging from $84.2 \%$ (DPA, krill oil diet, male rats) to $99.6 \%$ ( $\alpha$-linolenic acid (ALA) and EPA, krill oil diet, female rats), with the values for most of the fatty acids being in excess of $98 \%$. The largest differences in terms of both diet and sex were observed for DPA, where digestibility of this fatty acid was lowest in male rats fed the krill oil diet when compared with males fed the fish oil diet. DHA digestibility was not affected by the diet, whereas EPA digestibility was affected by the diet, but with opposite trend in the two sexes, and the differences were numerically small varying only from $99 \cdot 0$ to $99 \cdot 6 \%$. 
Table 2. The effect of fish oil and krill oil diets on the lipid content over the 6-week period of the experiment (Mean values and standard deviations of six cages per treatment; $n 6, N 24$ )

\begin{tabular}{|c|c|c|c|c|c|c|c|c|c|c|c|}
\hline \multirow[b]{3}{*}{ Lipid content } & \multicolumn{4}{|c|}{ Female rats } & \multicolumn{4}{|c|}{ Male rats } & \multirow[b]{3}{*}{$P_{\text {(Diet) }}$} & \multirow[b]{3}{*}{$P_{(\text {Sex })}$} & \multirow[b]{3}{*}{$P_{(\text {Diet } \times \text { Sex })}$} \\
\hline & \multicolumn{2}{|c|}{ Krill oil } & \multicolumn{2}{|c|}{ Fish oil } & \multicolumn{2}{|c|}{ Krill oil } & \multicolumn{2}{|c|}{ Fish oil } & & & \\
\hline & Mean & $\mathrm{SD}$ & Mean & $\mathrm{SD}$ & Mean & SD & Mean & $\mathrm{SD}$ & & & \\
\hline Whole body (mg/g) & 149 & 32 & 152 & 28 & 140 & 25 & 119 & 13 & NS & NS & NS \\
\hline \multicolumn{12}{|l|}{ Tissues $(\mathrm{mg} / \mathrm{g})$} \\
\hline Liver & 68 & 14 & 61 & 6 & 49 & 7 & 51 & 6 & NS & 0.001 & NS \\
\hline Heart & 31 & 1 & 29 & 3 & 27 & 2 & 29 & 1 & NS & 0.029 & NS \\
\hline Perirenal & 778 & 33 & 784 & 38 & 886 & 22 & 893 & 27 & NS & NS & NS \\
\hline White gastrocnemius & 25 & 5 & 24 & 3 & 22 & 2 & 20 & 3 & NS & 0.041 & NS \\
\hline
\end{tabular}

Table 3. The effect of fish oil and krill oil diets on apparent digestibility (\%) of lipids and $n$-3 PUFA (Mean values and standard deviations of six cages per treatment; $n 6, N 24$ )

\begin{tabular}{|c|c|c|c|c|c|c|c|c|c|c|c|}
\hline & \multicolumn{4}{|c|}{ Female rats } & \multicolumn{4}{|c|}{ Male rats } & \multirow[b]{3}{*}{$P_{(\text {Diet })}$} & \multirow[b]{3}{*}{$P_{(\text {Sex })}$} & \multirow[b]{3}{*}{$P_{(\text {Diet } \times \text { Sex })}$} \\
\hline & \multicolumn{2}{|c|}{ Krill oil } & \multicolumn{2}{|c|}{ Fish oil } & \multicolumn{2}{|c|}{ Krill oil } & \multicolumn{2}{|c|}{ Fish oil } & & & \\
\hline & Mean & $\mathrm{SD}$ & Mean & SD & Mean & $\mathrm{SD}$ & Mean & SD & & & \\
\hline Total lipid $n$-3 PUFA & $96 \cdot 8$ & 0.2 & $96 \cdot 8$ & 0.1 & $96 \cdot 3$ & 0.5 & $96 \cdot 7$ & 0.3 & NS & 0.016 & NS \\
\hline $18: 3 n-3$ (ALA) & $99 \cdot 6$ & 0.1 & $99 \cdot 8$ & 0.1 & $99 \cdot 4$ & 0.2 & $99 \cdot 7$ & 0.1 & 0.000 & 0.003 & NS \\
\hline$n-3$ LC-PUFA & & & & & & & & & & & \\
\hline $20: 5 n-3$ (EPA) & 99.6 & 0.9 & $99 \cdot 4$ & 0.7 & $99 \cdot 0$ & 0.1 & $99 \cdot 4$ & 0.0 & 0.000 & 0.000 & 0.000 \\
\hline 22 : $5 n-3$ (DPA) & $93 \cdot 3$ & $2 \cdot 3$ & $97 \cdot 1$ & $3 \cdot 2$ & $84 \cdot 2$ & 8.4 & $96 \cdot 9$ & 1.0 & 0.000 & 0.024 & 0.030 \\
\hline $22: 6 n-3$ (DHA) & $99 \cdot 3$ & 0.2 & $98 \cdot 8$ & 0.3 & 98.5 & 0.4 & $98 \cdot 8$ & 0.5 & NS & 0.016 & 0.021 \\
\hline
\end{tabular}

ALA, a-linolenic acid; LC-PUFA, long-chain PUFA.

\section{Fatty acid concentration of the rat whole body and tissues}

As shown in Table 4, the whole-body fatty acid concentration in female rats was significantly higher for all $n$-3 LC-PUFA compared with male rats at the end of experiment $(P=0 \cdot 000)$. The whole-body EPA concentration was similar in rats fed either fish oil diet or krill oil diet, for both sexes. However, DPA and DHA concentrations were significantly higher in the fish oil-fed group compared with the krill oil-fed group for both sexes at the end of the study period $(P=0.022)$. Supplementation with $n-3$ diets (fish oil and krill oil) led to a significant increase in whole-body $n-3$ LC-PUFA concentration compared with the baseline data over the 6-week period of the experiment (data not shown).

In terms of tissue concentrations, there were significant sex differences in the EPA and DHA concentrations (mg/g tissue) in the liver, heart and adipose tissue, but not muscle tissue, with tissues from female rats having a higher level of these two fatty acids compared with male rats (Table 4). There were no effects of dietary treatment on EPA and DHA levels in any of these four tissues.

Female rats had significantly higher concentrations of DPA than male rats in the liver and heart tissue, but not in muscle or adipose tissue. Interestingly, there were significantly higher concentrations of DPA in the liver and muscle tissue in krill oil-fed rats compared with fish oil-fed rats, but this difference was not observed in the heart or adipose tissue. These data suggest tissue-specific modulations of DPA metabolism, and are consistent with data from the recent study on male SpragueDawley rats, showing that an increased level of DPA was recorded in both the liver and the heart $2 \mathrm{~d}$ after the feeding of DPA ceased ${ }^{(30)}$.

\section{Eicosapentaenoic acid, docosapentaenoic acid and docosahexaenoic acid: mass balance}

Intake and excretion. Male rats consumed significantly more $n-3$ LC-PUFA (EPA, DPA and DHA) compared with female rats and excreted significantly more $n-3$ LC-PUFA (EPA, DPA and DHA). Between diets, $n$-3 LC-PUFA intake was higher in rats fed fish oil than rats fed krill oil; however, no significant differences in faecal excretion were observed.

Accumulation. The main whole-body $n$-3 LC-PUFA to be accumulated in both diet groups over the 6 weeks of the experiment was DHA (280-360 mg/animal), approximately four times more than DPA and EPA (70-90 mg/animal). In terms of net accumulation, significantly more DHA and total $n$ - 3 LC-PUFA accumulated in male rats compared with females, with no effect of diet observed (online Supplementary Table S2).

Appearance/disappearance. There was a net disappearance of all $n$-3 LC-PUFA in the experiment (intake greater than accumulation) in both the diet groups and for both sexes, except for DPA in male and female rats fed krill oil, where a small net appearance was observed (online Supplementary Table S2). The differences in the net disappearance of all $n-3$ 
Table 4. Selected whole-body and tissue fatty acids ( $\mathrm{mg} / \mathrm{g}$ tissue) of rats fed either krill oil or fish oil during the 6-week period of the experiment in both sexes

(Mean values and standard deviations of six cages per treatment; $n 6, N 24$ )

\begin{tabular}{|c|c|c|c|c|c|c|c|c|c|c|c|}
\hline & \multicolumn{4}{|c|}{ Female rats } & \multicolumn{4}{|c|}{ Male rats } & \multirow[b]{3}{*}{$P_{\text {(Diet) }}$} & \multirow[b]{3}{*}{$P_{(\text {Sex })}$} & \multirow[b]{3}{*}{$P_{\text {(Diet } \times \text { Sex })}$} \\
\hline & \multicolumn{2}{|c|}{ Krill oil } & \multicolumn{2}{|c|}{ Fish oil } & \multicolumn{2}{|c|}{ Krill oil } & \multicolumn{2}{|c|}{ Fish oil } & & & \\
\hline & Mean & SD & Mean & SD & Mean & SD & Mean & SD & & & \\
\hline \multicolumn{12}{|l|}{ Whole body } \\
\hline $20: 5 n-3(\mathrm{EPA})$ & 0.24 & 0.05 & 0.29 & 0.05 & 0.17 & 0.02 & 0.16 & 0.02 & NS & 0.000 & 0.039 \\
\hline $22: 5 n-3$ (DPA) & 0.26 & 0.07 & 0.32 & 0.05 & 0.21 & 0.02 & 0.22 & 0.02 & 0.013 & 0.000 & 0.043 \\
\hline $22: 6 n-3(\mathrm{DHA})$ & 1.01 & 0.08 & $1 \cdot 11$ & 0.15 & 0.80 & 0.06 & 0.88 & 0.06 & 0.003 & 0.000 & NS \\
\hline \multicolumn{12}{|l|}{ Liver } \\
\hline $20: 5 n-3$ (EPA) & 0.69 & 0.14 & 0.86 & 0.17 & 0.57 & 0.16 & 0.31 & 0.04 & NS & 0.000 & 0.001 \\
\hline $22: 5 n-3$ (DPA) & 0.39 & 0.18 & 0.24 & 0.07 & 0.26 & 0.04 & 0.14 & 0.02 & 0.004 & 0.008 & NS \\
\hline $22: 6 n-3(\mathrm{DHA})$ & $2 \cdot 26$ & 0.30 & 2.01 & 0.62 & 1.77 & 0.22 & 1.47 & 0.32 & NS & 0.005 & NS \\
\hline \multicolumn{12}{|l|}{ Heart } \\
\hline $20: 5 n-3$ (EPA) & 0.16 & 0.02 & 0.18 & 0.02 & 0.10 & 0.07 & 0.09 & 0.04 & NS & 0.001 & NS \\
\hline $22: 5 n-3$ (DPA) & 0.34 & 0.04 & 0.29 & 0.04 & 0.16 & 0.13 & 0.21 & $0 \cdot 10$ & NS & 0.002 & NS \\
\hline $22: 6 n-3(\mathrm{DHA})$ & 1.78 & 0.14 & 1.90 & 0.12 & 0.97 & 0.77 & 1.55 & 0.76 & NS & 0.018 & NS \\
\hline \multicolumn{12}{|c|}{ White gastrocnemius muscle } \\
\hline $20: 5 n-3$ (EPA) & $0 \cdot 18$ & 0.03 & 0.13 & 0.04 & 0.13 & 0.03 & 0.14 & 0.04 & NS & NS & 0.042 \\
\hline $22: 5 n-3$ (DPA) & 0.20 & 0.02 & 0.14 & 0.03 & 0.20 & 0.03 & 0.15 & 0.02 & 0.000 & NS & NS \\
\hline $22: 6 n-3(\mathrm{DHA})$ & 1.04 & 0.11 & 0.82 & 0.23 & 0.94 & 0.08 & 0.92 & $0 \cdot 10$ & NS & NS & NS \\
\hline \multicolumn{12}{|l|}{ Perirenal adipose } \\
\hline $20: 5 n-3$ (EPA) & 0.15 & 0.03 & 0.09 & 0.02 & 0.06 & 0.01 & 0.06 & 0.02 & NS & 0.001 & NS \\
\hline $22: 5 n-3$ (DPA) & 0.15 & 0.04 & 0.12 & 0.02 & 0.10 & 0.02 & 0.12 & 0.06 & NS & NS & NS \\
\hline $22: 6 n-3(\mathrm{DHA})$ & 0.17 & 0.05 & 0.15 & 0.04 & 0.09 & 0.03 & 0.09 & 0.03 & NS & 0.000 & NS \\
\hline
\end{tabular}

LC-PUFA were observed as a result of sex (male more than female, $P=0.000$ ) and diet (fish oil more than krill oil, $P=0 \cdot 000)$.

\section{Eicosapentaenoic acid, docosapentaenoic acid and docosahexaenoic acid apparent in vivo metabolism}

The whole-body method allowed for the calculation of apparent in vivo fatty acid metabolism (excretion, $\beta$-oxidation, bioconversion or deposition) as shown in Table 5 . The two most important pathways identified for the $n$-3 LC-PUFA in this study were $\beta$-oxidation and deposition, which together accounted for $>90 \%$ of the apparent in vivo metabolism (Table 5).

The main fate of EPA and DHA was $\beta$-oxidation; for EPA it was $93-95 \%$, whereas for DHA it ranged between 63 and $69 \%$. There were significant diet effects for DHA $\beta$-oxidation, which was higher in rats fed fish oil than those fed krill oil $(P=0.002)$. Sex did not significantly affect DHA metabolism (Table 5).

Apart from $\beta$-oxidation, deposition was the next main fate of EPA and DHA. In the case of EPA, deposition amounted to $<6 \%$ of the total metabolic activity, whereas in the case of DHA deposition accounted for between 29 and $36 \%$ (Table 5).

For EPA and DHA, there were very similar trends between diets and sex; however, this was not the case for DPA. The main fate of DPA differed between the diet groups. In the case of the fish oil groups, most of the DPA was $\beta$-oxidised ( 57 and $67 \%$ for female and male rats, respectively). In the krill oil groups, however, the majority of DPA was deposited (82-83\%), which contrasts with only $30-40 \%$ of the DPA being deposited in the fish oil groups. There was no significant difference in DPA deposition between sexes. DPA was not further bioconverted to other $n$-3 LC-PUFA (namely, 24:5n-3, $24: 6 n$-3 or DHA).
The apparent in vivo enzyme activities (expressed as millimoles of product per gram of BW per day) are reported in Table 6. The apparent total elongase activity and total desaturase activities ( $\Delta 5$-desaturase, $\Delta 6$-desaturase and $\Delta$ 9-desaturase) were significantly greater in rats fed krill oil-supplemented diets compared with those fed fish oil-supplemented diets. Apparent enzyme activities were also higher in male rats compared with female rats, with the exception of $\Delta$ 9-desaturase, where no significant difference was found.

\section{Discussion}

The aim of the present study was to compare the apparent 'bioavailability' of $n-3$ LC-PUFA originating from krill oil (PL-rich) $v$. that from fish oil (TAG-rich) in both male and female rats. It was planned to feed identical amounts $(1.5 \mathrm{mg} / \mathrm{g})$ of $n-3$ LC-PUFA in each diet; however, once the diet had been manufactured and tested for $n$-3 LC-PUFA content, it was found that the amounts were similar, but not identical $(1.61 v .1 .38 \mathrm{mg} / \mathrm{g}$, respectively). These are relatively small variations, normally encountered when formulating experimental diets, but for accuracy they have to be noted. Nevertheless, using the wholebody fatty acid balance method, the difference in $n-3$ LC-PUFA intake was not influential as it is possible to express the results as a percentage of the total intake of $n$-3 LC-PUFA (see Table 5).

During the 6 weeks of the trial, no mortality was recorded, experimental diets were highly palatable and all animals grew well. Lipid deposition in animals' whole body and individual tissues was not affected by the dietary treatment. Accordingly, previous studies have reported no significant difference in BW gain in mice ${ }^{(31)}$ or rats $^{(7,32)}$ fed either krill oil or fish oil. In the present study, the only differences observed in performance, 
Table 5. Fate of $n-3$ long-chain PUFA and EPA + DPA + DHA (\% of total intake) in rats fed either fish oil or krill oil diets during the 6-week experiment (Mean values and standard deviations of six cages per treatment; $n 6, N 24$ )

\begin{tabular}{|c|c|c|c|c|c|c|c|c|c|c|c|}
\hline & \multicolumn{4}{|c|}{ Female rats } & \multicolumn{4}{|c|}{ Male rats } & \multirow[b]{3}{*}{$P_{(\text {Diet })}$} & \multirow[b]{3}{*}{$P_{(\text {Sex })}$} & \multirow[b]{3}{*}{$P_{(\text {Diet } \times \text { Sex })}$} \\
\hline & \multicolumn{2}{|c|}{ Krill oil } & \multicolumn{2}{|c|}{ Fish oil } & \multicolumn{2}{|c|}{ Krill oil } & \multicolumn{2}{|c|}{ Fish oil } & & & \\
\hline & Mean & SD & Mean & SD & Mean & SD & Mean & SD & & & \\
\hline \multicolumn{12}{|l|}{ Excreted } \\
\hline $20: 5 n-3$ (EPA) & 0.7 & 0.0 & 0.6 & 0.0 & 0.9 & 0.0 & 0.5 & 0.0 & 0.011 & 0.000 & 0.000 \\
\hline $22: 5 n-3$ (DPA) & $6 \cdot 3$ & $2 \cdot 2$ & $2 \cdot 9$ & 0.3 & 14.9 & 7.9 & $3 \cdot 2$ & $1 \cdot 0$ & 0.000 & 0.025 & 0.031 \\
\hline $22: 6 n-3$ (DHA) & 0.7 & 0.2 & $1 \cdot 2$ & 0.3 & 1.5 & 0.4 & $1 \cdot 2$ & 0.5 & NS & 0.016 & 0.021 \\
\hline \multicolumn{12}{|l|}{$\beta$-oxidised } \\
\hline $20: 5 n-3(\mathrm{EPA})$ & 93.0 & $2 \cdot 4$ & 93.4 & $1 \cdot 2$ & 93.89 & 0.8 & $95 \cdot 6$ & 0.5 & NS & 0.017 & NS \\
\hline $22: 5 n-3$ (DPA) & $10 \cdot 6$ & $13 \cdot 1$ & 57.4 & $9 \cdot 6$ & $2 \cdot 7$ & 6.5 & $66 \cdot 6$ & 3.6 & 0.000 & NS & 0.030 \\
\hline $22: 6 n-3(\mathrm{DHA})$ & 63.5 & 4.2 & 68.6 & $5 \cdot 1$ & 63.9 & 2.4 & 69.9 & 2.4 & 0.002 & NS & NS \\
\hline $\mathrm{EPA}+\mathrm{DPA}+\mathrm{DHA}$ & $80 \cdot 7$ & $2 \cdot 7$ & $82 \cdot 0$ & $2 \cdot 8$ & $81 \cdot 2$ & 1.4 & 84.4 & $1 \cdot 2$ & 0.018 & NS & NS \\
\hline \multicolumn{12}{|l|}{ Bioconverted } \\
\hline $20: 5 n-3$ (EPA) & 0.7 & 0.2 & \multirow{2}{*}{\multicolumn{2}{|c|}{$\begin{array}{l}\text { ND } \\
\text { ND }\end{array}$}} & 0.6 & 0.4 & \multicolumn{2}{|c|}{ ND } & 0.017 & NS & NS \\
\hline $22: 5 n-3$ (DPA) & \multicolumn{2}{|c|}{ ND } & & & \multicolumn{2}{|c|}{ ND } & \multicolumn{2}{|c|}{ ND } & - & - & - \\
\hline$E P A+D P A$ & 0.5 & $0 \cdot 1$ & \multicolumn{2}{|c|}{ ND } & 0.4 & 0.2 & \multicolumn{2}{|c|}{ ND } & 0.17 & NS & NS \\
\hline \multicolumn{12}{|l|}{ Deposited } \\
\hline $20: 5 n-3$ (EPA) & $5 \cdot 8$ & 1.2 & 5.9 & $1 \cdot 1$ & 4.5 & 0.56 & 3.7 & 0.5 & NS & 0.000 & NS \\
\hline $22: 5 n-3$ (DPA) & 83.01 & $12 \cdot 3$ & 39.6 & $7 \cdot 2$ & $82 \cdot 3$ & 8.0 & $30 \cdot 2$ & 3.8 & 0.000 & NS & NS \\
\hline $22: 6 n-3(\mathrm{DHA})$ & $35 \cdot 7$ & $4 \cdot 1$ & $30 \cdot 2$ & $5 \cdot 0$ & $34 \cdot 4$ & 2.5 & 28.78 & $2 \cdot 4$ & 0.001 & NS & NS \\
\hline $\mathrm{EPA}+\mathrm{DPA}+\mathrm{DHA}$ & $18 \cdot 1$ & $2 \cdot 2$ & $17 \cdot 0$ & $2 \cdot 7$ & $16 \cdot 9$ & $1 \cdot 1$ & 14.5 & 1.2 & 0.036 & 0.027 & NS \\
\hline
\end{tabular}

ND, not detected.

Table 6. The apparent in vivo enzyme activities ( $\mathrm{mmol} / \mathrm{g}$ per $\mathrm{d}$ ) in rats fed either fish oil or krill oil diets during the 6-week experiment

(Mean values and standard deviations of six cages per treatment; $n 6, N 24$ )

\begin{tabular}{|c|c|c|c|c|c|c|c|c|c|c|c|}
\hline & \multicolumn{4}{|c|}{ Female } & \multicolumn{4}{|c|}{ Male } & \multirow[b]{3}{*}{$P_{\text {(Diet) }}$} & \multirow[b]{3}{*}{$P_{(\text {Sex })}$} & \multirow[b]{3}{*}{$P_{(\text {Diet } \times \text { Sex })}$} \\
\hline & \multicolumn{2}{|c|}{ Krill oil } & \multicolumn{2}{|c|}{ Fish oil } & \multicolumn{2}{|c|}{ Krill oil } & \multicolumn{2}{|c|}{ Fish oil } & & & \\
\hline & Mean & $\mathrm{SD}$ & Mean & $\mathrm{SD}$ & Mean & $\mathrm{SD}$ & Mean & $\mathrm{SD}$ & & & \\
\hline \multicolumn{12}{|l|}{ Elongation } \\
\hline $18: 2 n-6-20: n-6$ & $3 \cdot 7$ & $1 \cdot 3$ & $4 \cdot 0$ & $1 \cdot 3$ & $5 \cdot 2$ & $1 \cdot 2$ & $5 \cdot 6$ & 0.7 & NS & 0.003 & NS \\
\hline $20: 2 n-6-22: 2 n-6$ & 1.8 & 0.3 & 0.61 & 0.06 & $2 \cdot 2$ & 0.2 & $2 \cdot 4$ & 0.4 & NS & 0.000 & NS \\
\hline $18: 3 n-6-20: 3 n-6$ & 41.4 & $6 \cdot 3$ & $24 \cdot 9$ & $10 \cdot 4$ & 64.9 & $5 \cdot 6$ & $64 \cdot 5$ & 4.6 & 0.008 & 0.000 & NS \\
\hline $20: 4 n-6-22: 4 n-6$ & \multicolumn{2}{|c|}{ ND } & 0.30 & 0.36 & 1.9 & 0.6 & 3.9 & 0.8 & 0.000 & 0.000 & NS \\
\hline $22: 4 n-6-24: 4 n-6$ & 0.03 & 0.07 & 0.18 & 0.21 & 1.4 & 0.6 & 1.9 & 0.7 & NS & 0.000 & NS \\
\hline $18: 3 n-3-20: 3 n-3$ & 0.40 & 0.23 & 0.42 & 0.28 & 0.86 & 0.37 & $2 \cdot 1$ & 0.3 & 0.000 & 0.000 & NS \\
\hline $20: 3 n-3-22: 3 n-3$ & \multicolumn{2}{|c|}{ ND } & \multicolumn{2}{|c|}{ ND } & \multicolumn{2}{|c|}{ ND } & \multicolumn{2}{|c|}{ ND } & - & - & - \\
\hline $18: 4 n-3-20: 4 n-3$ & \multicolumn{2}{|c|}{ ND } & \multicolumn{2}{|c|}{ ND } & \multicolumn{2}{|c|}{ ND } & \multicolumn{2}{|c|}{ ND } & - & - & - \\
\hline $20: 5 n-3-22: 5 n-3$ & 3.6 & $5 \cdot 6$ & \multicolumn{2}{|c|}{ ND } & $2 \cdot 7$ & $2 \cdot 1$ & \multicolumn{2}{|c|}{ ND } & 0.018 & NS & NS \\
\hline $22: 5 n-3-24: 5 n-3$ & \multicolumn{2}{|c|}{ ND } & \multicolumn{2}{|c|}{ ND } & \multicolumn{2}{|c|}{ ND } & \multicolumn{2}{|c|}{ ND } & - & - & - \\
\hline \multicolumn{12}{|l|}{$\Delta 9$-desaturase } \\
\hline $14: 0-14: 1 n-5$ & $17 \cdot 3$ & $11 \cdot 5$ & $23 \cdot 2$ & $9 \cdot 72$ & $18 \cdot 2$ & $10 \cdot 0$ & $15 \cdot 2$ & $3 \cdot 0$ & NS & NS & NS \\
\hline $16: 0-16: 1 n-7$ & $838 \cdot 3$ & $479 \cdot 7$ & 699 & 406 & 1010 & 490 & $359 \cdot 3$ & $131 \cdot 6$ & 0.027 & NS & NS \\
\hline $18: 0-18: 1 n-9$ & \multicolumn{2}{|c|}{ ND } & \multicolumn{2}{|c|}{ ND } & & & & & - & - & NS \\
\hline $20: 0-20: 1 n-11$ & $1 \cdot 7$ & 0.4 & & & $2 \cdot 2$ & 0.5 & & & 0.000 & NS & NS \\
\hline $22: 0-22: 1 n-13$ & & & & & & & & & - & - & NS \\
\hline$\Delta 6$-desaturase & & & & & & & & & & & \\
\hline $18: 2 n-6-18: 3 n-6$ & 38.9 & $7 \cdot 0$ & $10 \cdot 1$ & $10 \cdot 5$ & 62.9 & $5 \cdot 5$ & $45 \cdot 8$ & 4.5 & 0.000 & 0.000 & NS \\
\hline $24: 4 n-6-24: 5 n-6$ & 0.029 & 0.071 & 0.18 & 0.21 & 1.4 & 0.6 & 1.9 & 0.7 & NS & 0.000 & NS \\
\hline $18: 3 n-3-18: 4 n-3$ & & & & & & & & & - & - & - \\
\hline $24: 5 n-3-24: 6 n-3$ & & & & & & & & & - & - & - \\
\hline$\Delta 5$-desaturase & & & & & & & & & & & \\
\hline $20: 3 n-6-20: 4 n-6$ & $35 \cdot 6$ & $5 \cdot 5$ & $18 \cdot 3$ & $9 \cdot 5$ & 56.9 & $5 \cdot 2$ & $55 \cdot 8$ & $5 \cdot 3$ & 0.003 & 0.000 & 0.007 \\
\hline $20: 4 n-3-20: 5 n-3$ & & & & & & & & & - & - & - \\
\hline Chain shortening & & & & & & & & & & & \\
\hline $24: 5 n-6-22: 5 n-6$ & 0.029 & 0.071 & 0.18 & 0.21 & 1.4 & 0.6 & 1.9 & 0.7 & NS & 0.000 & NS \\
\hline $24: 6 n-3-22: 6 n-3$ & & & & & & & & & - & - & - \\
\hline
\end{tabular}

ND, not detected. 
food intake and lipid deposition were, as expected, relative to sex

The $n$-3 LC-PUFA (EPA, DPA and DHA) concentrations in the tissues analysed showed some statistically significant differences. In the liver, heart and perirenal adipose tissue, these differences were mainly affected by sex, with female animals recording higher $n$-3 LC-PUFA concentrations compared with males. For whole body, similar sex differences were observed, but also a statistically significant effect of the oil was observed for DHA content, with animals fed fish oil recording higher DHA concentrations than animals fed krill oil. Nevertheless, although the results obtained by simply comparing tissue FA profiles are interesting, they are admittedly of limited value towards achieving a better understanding of $n$-3 LC-PUFA 'bioavailability'. In fact, the dietary intake of $n$ - 3 LC-PUFA provided by the two diets were similar, but not identical, and differences between treatments observed could be partially due to dietary supply rather than due to actual differences in 'bioavailability'. Therefore, and as mentioned above, a much greater and more accurate understanding of the actual metabolic fate of $n$ - 3 LC-PUFA provided by different oils can be achieved by observing the results of the whole-body fatty acid balance method.

The $n$-3 LC-PUFA in the diet were differently absorbed between the fish oil and krill oil groups and between sexes (Table 3). Female animals recorded a higher apparent digestibility for total lipid, $n$ - 3 PUFA and $n-3$ LC-PUFA compared with males. A marked effect on DPA digestibility by sex was observed for krill oil, with relatively low apparent digestibility values recorded in male rats fed the krill oil diet. On the other hand, DHA digestibility was not affected by the treatment, and was highly absorbed ( $>98 \%$ ) by both males and females fed either diet. However, when $n$-3 LC-PUFA were considered together (EPA + DPA + DHA) and reported as percentage of excreted fatty acids relative to total intake (Table 5), no differences were observed relative to diet treatment. Sex was found to have a direct effect on absorption (bioavailability), with female animals recording a significantly lower excretion (greater 'bioavailability'). The excretion (expressed as percentage of the total dietary intake) of $n-3$ LC-PUFA in this study ranged from 0.6 to $0.9 \%$ in females and from 1.1 to $1.5 \%$ in males. Tou et al. $^{(7)}$ observed no significant difference in EPA digestibility from krill oil and fish oil sources; however, they did report a higher DHA digestibility in female rats fed salmon oil compared with those fed krill oil. It is important to note here that in both the present study and in that of Tou et al. ${ }^{(7)}$ the differences between sexes, although tested as statistically significant, were possibly of limited biological significance. Nevertheless, we are short of likely explanations as to why sex can affect $n$ - 3 LC-PUFA absorption, and the present results warrant further specifically tailored studies.

Once absorbed, the metabolic fate of $n-3$ LC-PUFA was significantly affected by diet and sex. Metabolic fate here refers to whether the $n$-3 LC-PUFA were deposited (anabolism) or were $\beta$-oxidised (catabolism). Deposited here refers to whether the $n$-3 LC-PUFA were deposited as such or were bioconverted by desaturation or chain elongation.

A first important observation was that the major metabolic fate of $n$-3 LC-PUFA was towards catabolism ( $\beta$-oxidation), accounting for over $80 \%$ of total dietary intake, whereas deposition accounted for only 14.5 to $18.6 \%$ of the total dietary intake. It was found that for both sexes, significantly more $n-3$ LC-PUFA were $\beta$-oxidised when originating from fish oil compared with krill oil, with the average difference being $2 \cdot 8 \%$. Conversely, it was found that significantly more $n$-3 LC-PUFA were deposited when originating from krill oil than from fish oil in both sexes by a mean difference of $13.2 \%$. Furthermore, and independent of the dietary treatment, it was found that female rats deposited a significantly higher proportion of the dietary $n-3$ LC-PUFA compared with male rats, with the mean difference between females and males being $10 \cdot 3 \%$. Therefore, the present study suggests the existence of sex-specific differences with respect to $n$-3 LC-PUFA $\beta$-oxidation (higher in males) and deposition (higher in females), which when coupled with the known higher $n-3$ fatty acid bioconversion capability in females ${ }^{(16-18)}$ can explain the commonly reported higher $n-3$ status in females compared with males ${ }^{(13-15)}$, and possibly due to the overall higher $n$-3 LC-PUFA requirements of females during pregnancy and lactation.

Based on these data, it can be said that in this 6-week study a higher proportion of the $n$ - 3 LC-PUFA from krill oil were deposited in rat tissues (whole body) compared with $n$-3 LCPUFA originating from fish oil. This difference between deposition of $n$-3 LC-PUFA from krill oil and fish oil (13\%) was not of the order of the differences advertised in the marketing campaigns, which indicated the 'bioavailability' of krill oil being three to nine times higher than fish oil. Importantly, in the present study, it was also clearly shown that the 'bioavailability' is in actuality the same, but the resultant metabolic fate is different.

To our knowledge, this is the first study to report the wholebody bioavailability of $n$-3 LC-PUFA from either fish oil or krill oil in the rat. A number of previous studies by Cunnane's group, using the whole-body fatty acid balance method, examined the accumulation and apparent $\beta$-oxidation of shorter-chain PUFA in rats (linoleic acid and ALA). Generally, these studies reported a very high proportion of these PUFA being readily $\beta$-oxidised in pregnant rats ${ }^{(33)}$. Typically, studies on krill oil in rodents have looked at n-3 LC-PUFA levels in a number of tissues and compared $n$ - 3 LC-PUFA levels in these tissues with rats fed fish oil. For example, in a rat study by Batetta et al. ${ }^{(5)}$, EPA and DHA concentrations in the PL fraction of the liver and heart tissues were significantly increased in the krill oil-fed rats compared with fish oil-fed rats. Di Marzo et al. ${ }^{(6)}$ reported that a significantly higher DHA level was found in brain PL of rats provided with krill oil compared with fish oil. In addition, some human studies reported that plasma ${ }^{(9,10)}$ and erythrocytes ${ }^{(10)}$ EPA and DHA proportions increased significantly in the krill oil group compared with the fish oil group.

One of the novel observations of this study was that a significantly higher proportion of $n$-3 LC-PUFA from dietary fish oil was $\beta$-oxidised compared with krill oil. A possible reason for this difference might relate to the fact that fish oil is basically composed of TAG molecules, whereas krill oil is often rich in PL, as was the case in this study (65\% PL). Accordingly, there is now emerging evidence from animal and human studies showing that, following digestion and absorption, the $n-3$ LC-PUFA fatty acids from PL partition differently to those from TAG. 
Specifically, animal studies comparing essentially pure PL preparations with TAG forms are generally consistent in showing the different patterns of incorporation of $n-3$ LC-PUFA into chylomicron and plasma lipids or lipoprotein fractions. Amate et al. ${ }^{(34)}$ reported that arachidonic acid and DHA in TAG form partitioned preferentially into LDL-PL, whereas arachidonic acid and DHA in PL partitioned into HDL-PL. Rossmeisl et al. ${ }^{(35)}$ reported that EPA levels in the liver were nearly double in mice fed PL compared with the group fed TAG in a 9-week study in mice fed DHA plus EPA either in the TAG or PL form. Other data from the literature using either ${ }^{14} \mathrm{C}$ - or ${ }^{13} \mathrm{C}$-labelled DHA in PL or TAG form have reported differences in uptake of DHA into the brain ${ }^{(36)}$ or erythrocytes $^{(37,38)}$ in favour of the PL form. These examples emphasise that PL and TAG fatty acids are partitioned into tissue lipids in a different manner. It is, therefore, conceivable that the greater tissue incorporation of $n-3$ LC-PUFA fatty acids such as DHA from krill oil (PL) means that the DHA is less easily available to enzymatic pathways leading to $\beta$-oxidation than the $n-3$ LC-PUFA from fish oil (TAG).

Admittedly, there were a few limitations of this study that need to be reported. The first one was that the designed equal doses of $n$ - 3 LC-PUFA for fish oil and krill oil diets were not achieved during diet manufacturing (EPA + DHA + DPA levels of krill oil and fish oil were 1.38 and $1.61 \mathrm{mg} / \mathrm{g}$ of diet, respectively), despite several attempts in trial batches to get the doses equal, before final formulation and manufacturing. Nevertheless, to counteract this error, metabolic fates of each fatty acid were computed and expressed as a percentage of total intake, and thus were unaffected by the variation in dietary administration. The second one was that a longer-term trial would have been necessary to detect any changes of $n-3$ LC-PUFA status in other tissues such as brain ${ }^{(39)}$

In conclusion, this study showed that, in this 6-week study, a higher proportion of the dietary $n$-3 LC-PUFA, and specifically DPA and DHA but not EPA, from krill oil was deposited in rat tissues (whole body) compared with fish oil by about $13 \%$. However, and importantly, this study also demonstrated that this difference was not due to a difference in 'bioavailability' (absorption) but due to a difference in metabolic fate (anabolism $v$. catabolism). It was estimated that most of EPA (>90\%) and more than half of DHA (>60\%) were $\beta$-oxidised in both diet groups. Most of the DPA was $\beta$-oxidised ( 57 and $67 \%$ for female and male rats, respectively) in the fish oil group; however, for the krill oil group, the majority of DPA was deposited (82-83\%). Furthermore, a higher proportion of the dietary $n-3$ LC-PUFA was deposited in female rats (whole body) compared with male rats, by about $11 \%$, independent of diet. This study proved that the $\beta$-oxidation and deposition, but not absorption, of LC $n-3$ fatty acids was affected by the oil source (krill oil or fish oil) in the rat.

\section{Acknowledgements}

The authors thank Dr David Francis for the lipid classes analysis of the tested oils and James Emery for his technical support.

This study was supported by the Australian Research Council's Discovery Projects funding scheme (Project DP1093570). The views expressed here are those of the authors and are not necessarily those of the Australian Research Council. The Molecular Medicine Strategic Research Centre, Deakin University, is also acknowledged for the financial support. In addition, authors thank Swisse, Melbourne, Australia, for their kind donation of the oils. The above-mentioned funders had no role in the design, analysis or writing of this article.

All authors contributed to this research. S. G.: contributed to the study design, conducted the animal study, analysis of tissues and whole body of rats, conducted the statistical analysis and preparation of the manuscript. K. H.: contributed to the tissue and whole-body analysis and preparation of the manuscript. G. M. T.: contributed substantially to the conception and the experimental design, diet analysis, data analysis, interpretation of findings and provided critical revision of the article. A. J. S.: contributed substantially to the conception and the experimental design, interpretation of findings and provided critical revision of the article.

There are no conflicts of interest to declare.

\section{Supplementary material}

For supplementary material/s referred to in this article, please visit http://dx.doi.org/10.1017/S0007114515002457

\section{References}

1. Kaur G, Cameron-Smith D, Garg M, et al. (2011) Docosapentaenoic acid (22:5n-3): a review of its biological effects. Prog Lipid Res 50, 28-34.

2. Burri L, Hoem N, Banni S, et al. (2012) Marine omega-3 phospholipids: metabolism and biological activities. Int J Mol Sci 13, 15401-15419.

3. Harris WS, Mozaffarian D, Michael L et al. (2009) Towards establishing dietary reference intakes for eicosapentaenoic and docosahexaenoic acids. J Nutr 139, 804-819.

4. Tou JC, Jaczynski J \& Chen Y-C (2007) Krill for human consumption: nutritional value and potential health benefits. Nutr Rev 65, 63-77.

5. Batetta B, Griinari M, Carta G, et al. (2009) Endocannabinoids may mediate the ability of (n-3) fatty acids to reduce ectopic fat and inflammatory mediators in obese Zucker rats. $J$ Nutr 139, 1495-1501.

6. Di Marzo V, Griinari M, Carta G, et al. (2010) Dietary krill oil increases docosahexaenoic acid and reduces 2arachidonoylglycerol but not $\mathrm{N}$-acylethanolamine levels in the brain of obese Zucker rats. Int Dairy J 20, 231-235.

7. Tou JC, Altman SN, Gigliotti JC, et al. (2011) Different sources of omega-3 polyunsaturated fatty acids affects apparent digestibility, tissue deposition, and tissue oxidative stability in growing female rats. Lipids Health Dis 10, 179.

8. Maki KC, Reeves MS, Farmer M, et al. (2009) Krill oil supplementation increases plasma concentrations of eicosapentaenoic and docosahexaenoic acids in overweight and obese men and women. Nutr Res 29, 609-615.

9. Ulven S, Kirkhus B, Lamglait A, et al. (2011) Metabolic effects of krill oil are essentially similar to those of fish oil but at lower dose of EPA and DHA, in healthy volunteers. Lipids 46, 37-46.

10. Ramprasath VR, Eyal I, Zchut S, et al. (2013) Enhanced increase of omega-3 index in healthy individuals with response to 4-week n-3 fatty acid supplementation from krill oil versus fish oil. Lipids Heath Dis 12, 178. 
11. Laidlaw M, Cockerline CA \& Rowe WJ (2014) A randomized clinical trial to determine the efficacy of manufacturers' recommended doses of omega-3 fatty acids from different sources in facilitating cardiovascular disease risk reduction. Lipids Health Dis 13, 99.

12. Schuchardt JP, Schneider I, Meyer H, et al. (2011) Incorporation of EPA and DHA into plasma phospholipids in response to different omega-3 fatty acid formulations - a comparative bioavailability study of fish oil vs. krill oil. Lipids Health Dis 10, 145.

13. Lohner S, Fekete K, Marosvolgyi T, et al. (2013) Gender differences in the long-chain polyunsaturated fatty acid status: systematic review of 51 publications. Ann Nutr Metab 62, 98-112.

14. Sarter B, Kelsey KS, Schwartz TA, et al. (2015) Blood docosahexaenoic acid and eicosapentaenoic acid in vegans: associations with age and gender and effects of an algal-derived omega-3 fatty acid supplement. Clin Nutr 34, 212-218.

15. Howe PR, Buckley JD, Murphy KJ, et al. (2014) Relationship between erythrocyte omega-3 content and obesity is gender dependent. Nutrients 6, 1850-1860.

16. Kitson AP, Smith TL, Marks KA, et al. (2012) Tissue-specific sex differences in docosahexaenoic acid and $\Delta 6$-desaturase in rats fed a standard chow diet. Appl Physiol Nutr Metab 37, $1200-1211$

17. Extier A, Langelier B, Perruchot MH, et al. (2010) Gender affects liver desaturase expression in a rat model of $n-3$ fatty acid repletion. J Nutr Biochem 21, 180-187.

18. Burdge GC, Slater-Jefferies JL, Grant RA, et al. (2008) Sex, but not maternal protein or folic acid intake, determines the fatty acid composition of hepatic phospholipids, but not of triacylglycerol, in adult rats. Prostaglandins Leukot Essent Fatty Acids 78, 73-79.

19. Salem N Jr \& Kuratko CN (2014) A reexamination of krill oil bioavailability studies. Lipids Health Dis 13, 137.

20. Ghasemifard S, Turchini GM \& Sinclair AJ (2014) Review: omega-3 long chain fatty acid 'bioavailability': a review of evidence and methodological considerations. Prog Lipid Res 56, $92-108$.

21. Kwan KC (1997) Oral bioavailability and first-pass effects. Drug Metab Dispos 25, 1329-1336.

22. Turchini GM, Francis DS \& De Silva SS (2007) A whole body, in vivo, fatty acid balance method to quantify PUFA metabolism (desaturation, elongation and beta-oxidation). Lipids $\mathbf{4 2}$, 1065-1071.

23. Folch J, Lees M \& Stanley GHS (1957) A simple method for the isolation and purification of total lipids from animal tissues. J Biol Chem 226, 497-509.

24. Christie WW (2003) Lipid Analysis. Isolation, Separation, Identification and Structural Analysis of Lipids, 3rd ed. Bridgwater, UK: The Oily Press, PJ Barnes and Associates.

25. Norambuena F, Lewis M, Hamid NKA, et al. (2013) Fish oil replacement in current aquaculture feed: is cholesterol a hidden treasure for fish nutrition? PloS ONE 8, e81705.
26. Turchini GM \& Francis D (2009) Fatty Acid Metabolism (Desaturation, Elongation and $\beta$-Oxidation) in Rainbow Trout Fed Fish Oil- or Linseed Oil-Based Diets. Br J Nutr 102, 69-81.

27. Cunnane SC, Ryan MA, Craig KS, et al. (1995) Synthesis of linoleate and alpha-linolenate by chain elongation in the rat. Lipids 30, 781-783.

28. Cunnane SC \& Yang J (1995) Zinc deficiency impairs whole-body accumulation of polyunsaturates and increases the utilization of [1-14C]linoleate for de novo lipid synthesis in pregnant rats. Can J Physiol Pharmacol 73, $1246-1252$

29. Cunnane SC \& Anderson MJ (1997) The majority of dietary linoleate in growing rats is beta-oxidized or stored in visceral fat. J Nutr 127, 146-152.

30. Ghasemi Fard S, Linderborg KM, Turchini GM, et al. (2014) Comparison of the bioavailability of docosapentaenoic acid (DPA, 22:5n-3) and eicosapentaenoic acid (EPA, 20:5n-3) in the rat. Prostaglandins Leukot Essent Fatty Acids 90, 23-26.

31. Burri L, Berge K, Wibrand K, et al. (2011) Differential effects of krill oil and fish oil on the hepatic transcriptome in mice. Front Genet 2, 45.

32. Ferramosca A, Conte L \& Zara V (2012) A krill oil supplemented diet reduces the activities of the mitochondrial tricarboxylate carrier and of the cytosolic lipogenic enzymes in rats. J Anim Physiol Anim Nutr (Berl) 96, 295-306.

33. Cunnane SC \& Chen ZY (1995) Fasting/refeeding has little lasting effect on long-chain fatty acids accumulating in the developing rat conceptus. Biochim Biophys Acta 1255, 113-117.

34. Amate L, Gil A \& Ramirez M (2001) Feeding infant piglets formula with long-chain polyunsaturated fatty acids as triacylglycerols or phospholipids influences the distribution of these fatty acids in plasma lipoprotein fractions. J Nutr 131, $1250-1255$

35. Rossmeisl M, Jilkova ZM, Kuda O, et al. (2012) Metabolic effects of n-3 PUFA as phospholipids are superior to triglycerides in mice fed a high-fat diet: possible role of endocannabinoids. PLOS ONE 7, e38834.

36. Graf BA, Duchateau GS, Patterson AB, et al. (2010) Age dependent incorporation of 14C-DHA into rat brain and body tissues after dosing various 14C-DHA-esters. Prostaglandins Leukot Essent Fatty Acids 83, 89-96.

37. Lemaitre-Delaunay D, Pachiaudi C, Laville M, et al. (1999) Blood compartmental metabolism of docosahexaenoic acid (DHA) in humans after ingestion of a single dose of [(13)C] DHA in phosphatidylcholine. J Lipid Res 40, 1867-1874.

38. Brossard N, Croset M, Normand S, et al. (1997) Human plasma albumin transports [13C]docosahexaenoic acid in two lipid forms to blood cells. J Lipid Res 38, 1571-1582.

39. Sinclair A, Begg D, Mathai M, et al. (2007) Omega 3 Fatty Acids and the Brain: Review of Studies in Depression. Asia Pac J Clin Nutr 16, 391-397. 